\title{
New neighbours
}

\section{Spectroscopy of DENIS nearby stars candidates}

\author{
F. Crifo ${ }^{1}$, N. Phan-Bao ${ }^{2,8}$, X. Delfosse ${ }^{3}$, T. Forveille ${ }^{4}$, J. Guibert ${ }^{5}$, E. L. Martín ${ }^{6,7}$, and C. Reylé ${ }^{9}$ \\ 1 GEPI, Observatoire de Paris, 5 place J. Janssen, 92195 Meudon Cedex, France \\ e-mail: francoise.crifo@obspm.fr \\ 2 Institute of Astronomy and Astrophysics, Academia Sinica. PO Box 23-141, Taipei 106, Taiwan, ROC \\ 3 Laboratoire d'Astrophysique de Grenoble, Université J. Fourier, BP 53, 38041 Grenoble, France \\ ${ }^{4}$ Canada-France-Hawaii Telescope Corporation, 65-1238 Mamalahoa Highway, Kamuela, HI 96743 USA \\ 5 Centre d'Analyse des Images, GEPI, Observatoire de Paris, 61 avenue de l'Observatoire, 75014 Paris, France \\ ${ }^{6}$ Instituto de Astrofísica de Canarias, C/ Vía Láctea s/n, 38200 La Laguna (Tenerife), Spain \\ 7 University of Central Florida, Dept. of Physics, PO Box 162385, Orlando, FL 32816-2385, USA \\ ${ }^{8}$ University of Hue, Dept. of Physics, 32 Le Loi, Hue, Vietnam \\ 9 CNRS UMR 6091, Observatoire de Besançon, BP 15, 25010 Besançon Cedex, France
}

Received 5 March 2005 / Accepted 7 June 2005

\begin{abstract}
We present spectra of 36 nearby star candidates and 3 red giant candidates, identified in the DENIS database. 32 of the dwarf candidates are nearby red dwarfs, with spectral types from M 5.5 to M 8.5. Out of 11 targets with low proper motion $\left(\mu<0.1 \operatorname{arcsec} \mathrm{yr}^{-1}\right)$ but a Reduced Proper Motion above an inclusive threshold, 9 are red dwarfs. The 4 contaminants are all reddened F-K main sequence stars, and could have been eliminated by checking for small well-known high latitude molecular clouds. These stars might be of interest as probes of interstellar absorption. For the red dwarfs we derive spectral types and spectroscopic distances, using a new calibration of the PC 3 spectral index to absolute magnitudes in the $I, J, H$ and $K$ photometric bands.

We confirm 2 new members of the 12 pc volume ( 2 new M 8.5), and one M 7.5 NLTT object closer than 10 pc; and show that one quarter of the stars with photometric distances under $30 \mathrm{pc}$ have too small a proper motion for inclusion in the NLTT catalog.
\end{abstract}

Key words. stars: low-mass, brown dwarfs - solar neighbourhood - stars: fundamental parameters - stars: distances

\section{Introduction}

We present spectroscopic observations of candidate nearby red dwarfs stars $(d<30 \mathrm{pc} ; 2 \leq I-J \leq 3)$ from Phan-Bao et al. (2001, 2003, hereafter Papers I and II respectively). These late-M dwarf candidates were either found in the DENIS survey (Epchtein et al. 1997) over 5700 square degrees, or crossidentified over a wider area between the DENIS database and the LHS or NLTT catalogs (Luyten 1979, 1980). All were further selected by "Maximum Reduced Proper Motion" (hereafter MRPM, see Paper II). That selection method retrieves solar neighbourhood red dwarfs down to very low proper motions, $\mu<0.1 \operatorname{arcsec} \mathrm{yr}^{-1}$, well below the $0.18 \operatorname{arcsec} \mathrm{yr}^{-1}$ threshold of the NLTT catalog (Luyten 1979). Our current inventory of the immediate solar neighbourhood (particularly for faint members) relies heavily on the NLTT catalog and its predecessors, in spite of early efforts by Vyssotsky (1943, 1946, 1952) to correct this recognized kinematics bias through spectrophotometric selection. This makes low proper motion neighbours of particular interest, and we felt that a spectroscopic validation of our selection criteria was highly desirable.

Section 2 describes the sample and the spectroscopic observations. Section 3 presents the measurements of various spectroscopic indices and derives an improved calibration of the PC3 spectral index (Martín et al. 1999) to absolute $I, J$, and $K$ magnitudes. It then discusses the spectral types and distances of the confirmed late-M dwarfs. Section 4 discusses the contaminating giants and reddened earlier stars, examines the effectiveness of MRPM selection, and gives statistical elements about the number of low-proper motion stars in the solar neighbourhood. The final section summarizes our results.

\section{Data sample and spectroscopic observations}

\subsection{Data sample}

Papers I and II examine a total of 132 DENIS sources with $2 \leq I-J \leq 3$, and classify them into 80 probable dwarfs and 52 probable giants. The 52 giants and 62 of the dwarf 
candidates originate within 5700 square degrees, where we examined all DENIS sources within well defined colour and magnitude limits. They together constitute our main sample, which we use for statistical discussions. 18 dwarf candidates were picked outside that area, from a cross-identification of the DENIS database and the NLTT catalog. They constitute our extended sample, with a less direct statistical pedigree. The targets were divided into 3 priority groups, running from A (highest priority) to $\mathrm{C}$ (lowest):

- A: 11 probable red dwarfs with low proper motion (low$\mathrm{PM}), \mu<0.1 \operatorname{arcsec}^{-1}$ (from Table 3 of Paper II). Establishing their status and validating the selection method was our highest priority.

- B and b: 69 very likely red dwarfs with high proper motion (high-PM), $\mu>0.1$ arcsec $\mathrm{yr}^{-1}$ (from Table 5 of Paper I and Tables $3 \mathrm{a}$ and $3 \mathrm{~b}$ of Paper II). 51 of them (noted B) belong to the main sample, and 18 (noted $\mathrm{b}$ ) belong to the extended sample.

- C: 52 probable or previously known red giants (all with low-PM, $\mu<0.1 \operatorname{arcsec}_{\mathrm{yr}^{-1}}$ ).

The A, B and C stars constitute the main sample, while the $\mathrm{b}$ targets represent the extended sample. They were observed at ESO during two observing runs, on the T152 and NTT telescopes.

\subsection{Spectroscopic observations and reductions}

\subsubsection{T152 observations}

27 of the stars were observed with the Boller \& Chivens spectrograph mounted on the ESO $1.52 \mathrm{~m}$ telescope at La Silla on August 4th, 5th and 6th 2002, shortly before that telescope was closed on October 1st. The first night was cloudy and very windy, and we could only observe 2 targets, DENISP J0004-17 and DENIS-P J0013-00, with mediocre seeing (1".5). The two remaining nights were partly photometric and the seeing was around $1^{\prime \prime}$. The detector was La Silla CCD\#38 $(2688 \times 512$ pixels $)$ and we used grating\#5, giving a dispersion of $0.13 \mathrm{~nm}$ pixel $^{-1}$ and a wavelength coverage from $645 \mathrm{~nm}$ to $900 \mathrm{~nm}$. We used the $2^{\prime \prime}$ slit, which corresponds to a spectral resolution of $0.32 \mathrm{~nm}$ for the arc lines. The stellar images were narrower than the slit width, and the stellar spectra therefore have somewhat higher spectral resolution. The small telescope size and a maximum exposure time of $55 \mathrm{~min}$ prevented observation of the fainter targets. Low amplitude fringes are visible redwards of $760 \mathrm{~nm}$ in the raw spectra, but they flat-field out very well. We observed two spectrophotometric standards from the ESO list (available at ftp.eso.org/pub/stecf/standards/ctiostan/), EG 274 and LTT 7987.

We calibrated the spectra using standard IRAF packages: CCDPROC for bias-subtraction, flat-fielding and bad pixel correction, and APALL for wavelength and flux calibration, using He-Ar lamp spectra obtained at the beginning of each night and spectra of the spectrophotometric standards.

\subsubsection{NTT observations}

12 mostly fainter stars were observed at the NTT telescope in November 2003, using the EMMI instrument in its Red Imaging and Low-Dispersion mode (RILD). In this mode the dispersion is $0.36 \mathrm{~nm}$ pixel $^{-1}$, and the effective wavelength range is 520 to $950 \mathrm{~nm}$. The weather was photometric and the seeing varied from 0.5 to 1.5 arcsec. We selected the $1^{\prime \prime}$ slit, which corresponds to a spectral resolution of $1.04 \mathrm{~nm}$ for the arc lines. Exposure times ranged from $30 \mathrm{~s}$ to $1200 \mathrm{~s}$ depending on the target's magnitude. The spectrophotometric standards, LTT 2415 and Feige 110 were chosen from the ESO list. All reduction was performed within MIDAS.

One more object was observed: DENIS P-J0410480$125142=$ LP 714-37. But as it was seen double with a large separation on the NTT pointing image, it is presented in a separate paper (Phan-Bao et al. 2005). No other visual binaries were seen in the acquisition images.

\subsubsection{Analysis of the spectra}

All spectra were normalized to 1 over the $754-758 \mathrm{~nm}$ interval, the denominator of the PC3 index and a region with a good flat pseudo-continuum.

At the resolution of these spectra, the presence of the NaI and KI doublets and the absence of the CaII triplet immediately distinguish $\mathrm{M}$ dwarfs from $\mathrm{M}$ giants, as does the general shape of the TiO bands. To our surprise, a few of the targets also have much earlier spectra, reddened by intervening dust. One, J1625-24 (LP 862-26), had in fact been previously recognized as not an M dwarf (Reid 2003, quoting a private communication by S. Salim). Blue spectra would be needed for precise classification at these earlier spectral types, but the 4 stars are dwarfs of spectral type $\mathrm{F}$ to $\mathrm{K}$.

The 39 observed stars divide into:

- $32 \mathrm{M}$ dwarfs: 9 from group A, 18 from group B and 5 from group b;

- 3 giants, all from group C, as expected;

- 4 highly reddened distant main-sequence stars, 2 from group A and 1 each from groups B and $\mathrm{b}$.

We could observe all group A stars and therefore have a complete view of the low proper motion candidates.

Figures $1-3$ respectively show the 20 T152 spectra and 12 NTT spectra of nearby late-M dwarfs and the 7 T152 spectra of distant stars. We defer a discussion of the later objects to Sect. 4.

\section{Spectroscopic indices and spectral type classification}

In the past few years, a number of interesting spectroscopic indices have been defined for M and L dwarfs (e.g. Reid et al. 1995; Martín et al. 1999; Cruz \& Reid 2002). Lépine et al. (2003) provides a good summary and selects the most effective of those, which in combination allow to determine both spectral types and qualitative metallicities. Table 1 lists our measurements of the $\mathrm{PC} 3, \mathrm{TiO} 5, \mathrm{CaH} 2, \mathrm{CaH} 3, \mathrm{VO} 1, \mathrm{VO} 2, \mathrm{TiO} 6$ and 


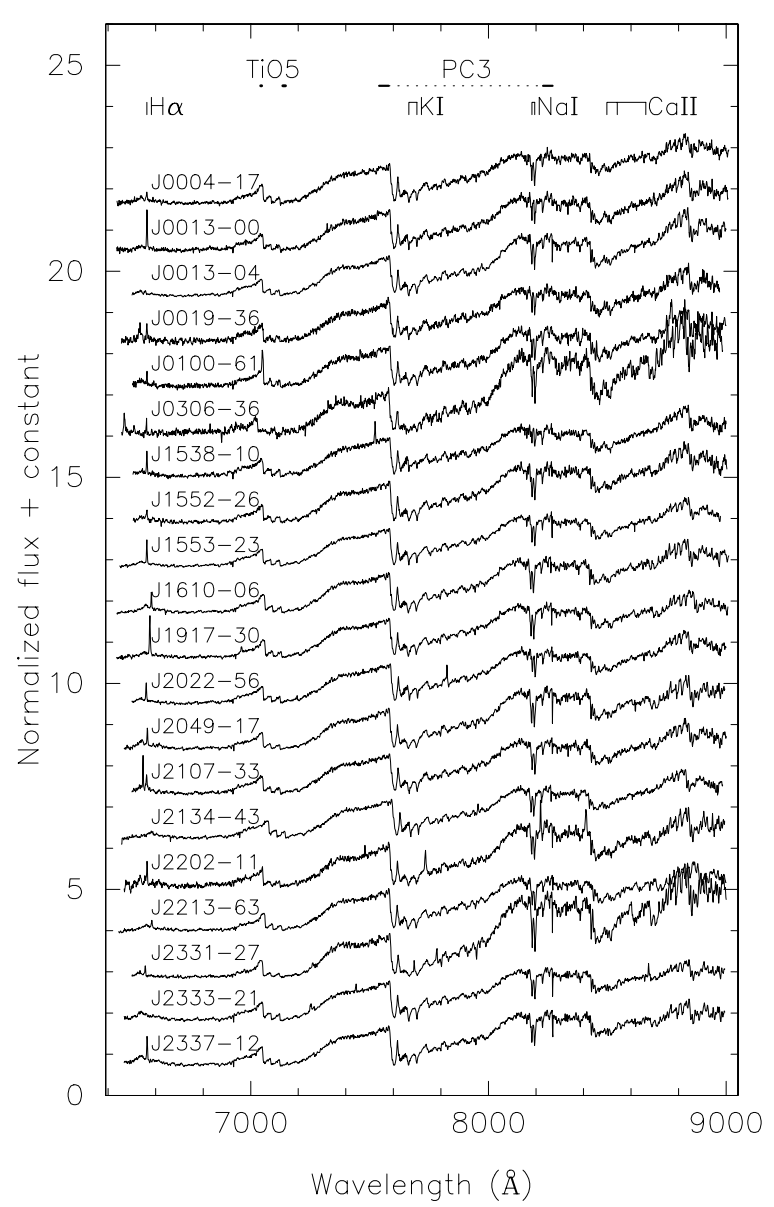

Fig. 1. Spectra of the $20 \mathrm{~T} 152$ late-M dwarfs. The positions of the $\mathrm{H}_{\alpha}$, $\mathrm{NaI}, \mathrm{KI}$ and CaII lines are indicated, as well as the spectral intervals used to compute the TiO5 and PC3 indices.

TiO7 spectroscopic indices for the $32 \mathrm{M}$ dwarfs. PC3 measures the slope of a pseudo-continuum, and all other indices measure molecular features. The low resolution of our NTT spectra biases their TiO5, $\mathrm{CaH} 2$ and $\mathrm{CaH} 3$ indices in the direction of earlier spectral types, and those measurements should thus be interpreted with caution.

Two of the indices, PC3 and TiO5, are of particular value for spectral type determination, while the others mostly provide information on the metallicity. PC3 and TiO5 have complementary strengths and weaknesses:

- The defining bands of PC3 (numerator: 823-827 nm, denominator: 754-758 nm, Martín et al. 1999) are wide, but separated by almost $80 \mathrm{~nm}$. The index is therefore easily measured with good signal to noise ratio, resistent to moderate changes of the spectral resolution, and insensitive to small errors in the wavelength calibration. It is on the other hand rather sensitive to an imperfect relative flux calibration. On the astrophysical side, PC3 is an excellent spectral type indicator over the [M 2.5, L1] range, and it increases monotonically over the full M class.

- The two defining bands of TiO5 (numerator: 712.6-713.5 nm, denominator: 704.2-704.6 nm, Reid et al. 1995; Cruz \& Reid 2002) are within less than $10 \mathrm{~nm}$, and the index is therefore resistent to flux calibration

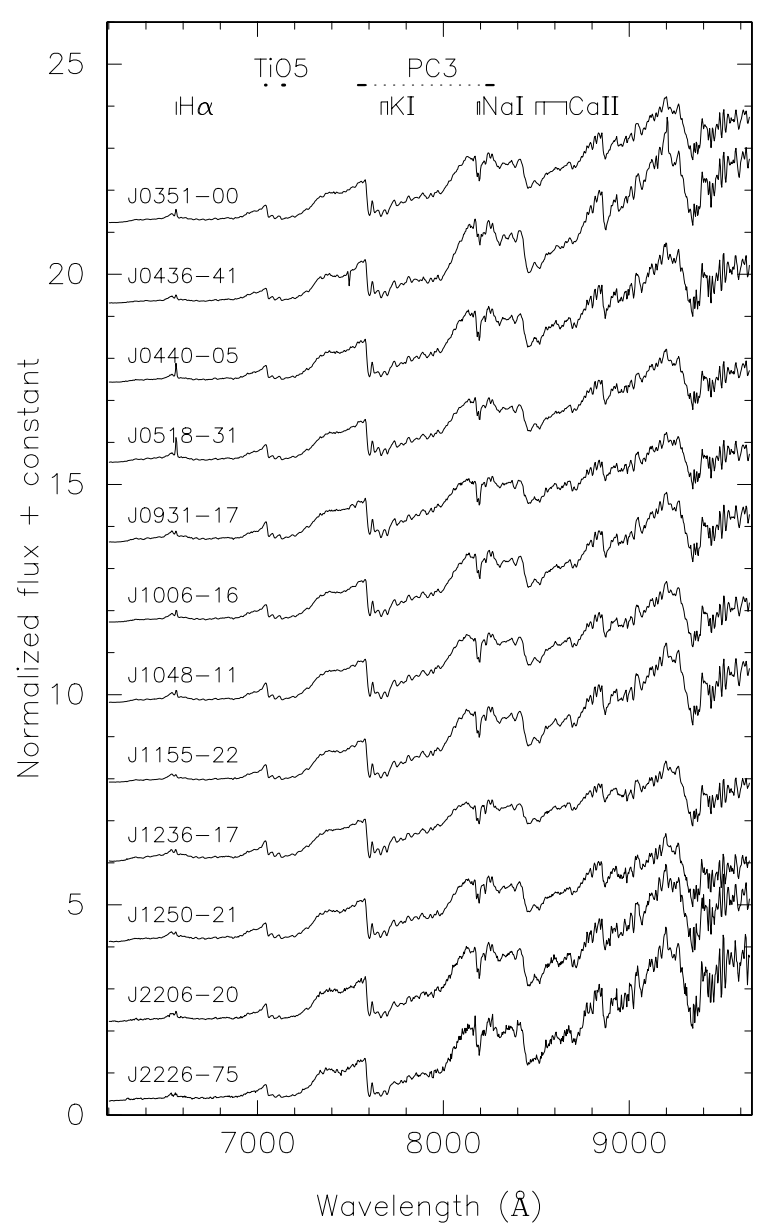

Fig. 2. Same as Fig. 1, for the 12 NTT spectra.

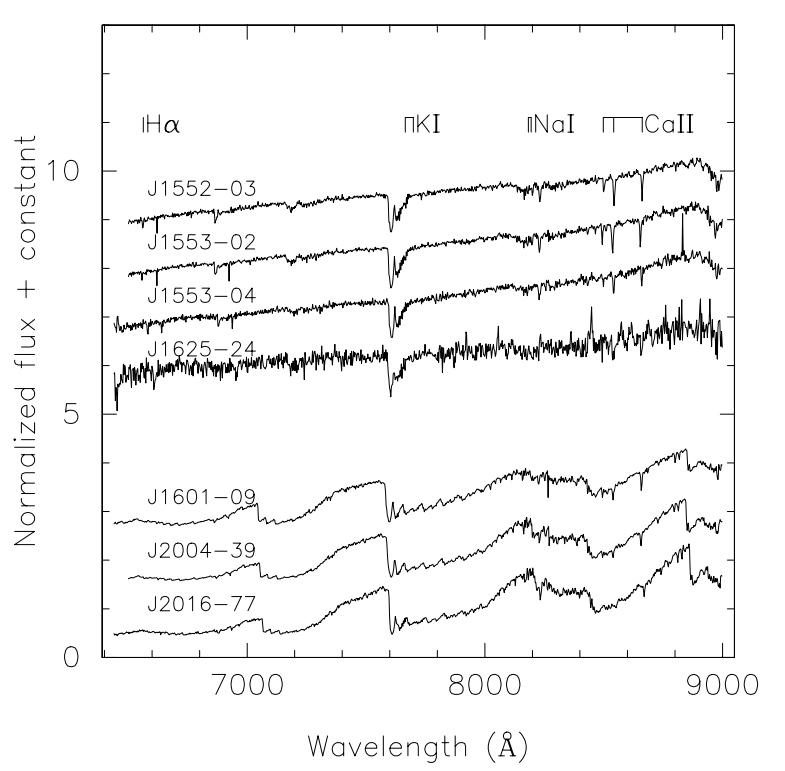

Fig. 3. Spectra of the three M giants rejected by the MRPM selection (bottom), and the four reddened early-type main-sequence stars (top).

errors. On the other hand, the interval for the denominator is narrow and positioned at the very head of a molecular band. The index is therefore sensitive to both spectral resolution changes and small errors in the wavelength 
Table 1. Spectral indices for the 32 nearby red dwarfs observed at T152 and NTT.

\begin{tabular}{|c|c|c|c|c|c|c|c|c|c|c|c|c|}
\hline Stars & $\begin{array}{l}\text { Other } \\
\text { name } \\
(2)\end{array}$ & PC3 & $\mathrm{TiO} 5$ & $\mathrm{CaH} 2$ & $\mathrm{CaH} 3$ & $\begin{array}{l}\text { VO1 } \\
\text { (7) }\end{array}$ & $\begin{array}{l}\mathrm{VO} 2 \\
(8)\end{array}$ & $\begin{array}{l}\text { TiO6 } \\
(9)\end{array}$ & $\begin{array}{l}\mathrm{TiO} 7 \\
(10)\end{array}$ & $\begin{array}{l}\text { SpT } \\
\text { PC3 } \\
(11)\end{array}$ & $\begin{array}{l}\mathrm{SpT} \\
\mathrm{TiO} 5 \\
(12)\end{array}$ & $\operatorname{Pr}$ \\
\hline \multicolumn{13}{|l|}{ T152 objects: } \\
\hline J0004575-170937 & $\ldots$ & 1.32 & 0.262 & 0.296 & 0.561 & 0.886 & 0.608 & 0.517 & 0.728 & M 5.5 & M 5.5 & B \\
\hline J0013093-002551 & $\ldots$ & 1.37 & 0.216 & 0.358 & 0.647 & 0.835 & 0.500 & 0.435 & 0.644 & M 5.5 & M 6.0 & $\mathrm{~A}$ \\
\hline J0013466-045736 & LHS 1042 & 1.48 & 0.228 & 0.287 & 0.635 & 0.807 & 0.463 & 0.463 & 0.609 & M 6.0 & M 5.5 & B \\
\hline J0019275-362015 & $\ldots$ & 1.35 & 0.229 & 0.273 & 0.629 & 0.818 & 0.541 & 0.459 & 0.667 & M 5.5 & M 5.5 & $\mathrm{~B}$ \\
\hline J0100021-615627 & $\ldots$ & 1.45 & 0.161 & 0.223 & 0.457 & 0.820 & 0.485 & 0.457 & 0.653 & M 6.0 & M 6.5 & A \\
\hline J0306115-364753 & $\ldots$ & 1.97 & 0.082 & 0.268 & 0.481 & 0.780 & 0.367 & 0.430 & 0.531 & M 8.5 & M7.0: & $\mathrm{B}$ \\
\hline $\mathrm{J} 1538317-103850$ & $\ldots$ & 1.25 & 0.241 & 0.313 & 0.634 & 0.837 & 0.552 & 0.486 & 0.659 & M 5.0 & M 5.5 & A \\
\hline $\mathrm{J} 1552446-262313$ & LP 860-41 & 1.42 & 0.230 & 0.264 & 0.567 & 0.827 & 0.543 & 0.480 & 0.670 & M 6.0 & M 5.5 & $\mathrm{~b}$ \\
\hline $\mathrm{J} 1553571-231152$ & LP 860-46 & 1.30 & 0.283 & 0.330 & 0.644 & 0.844 & 0.565 & 0.476 & 0.706 & M 5.0 & M 5.0 & $\mathrm{~b}$ \\
\hline $\mathrm{J} 1610584-063132$ & LP 684-33 & 1.36 & 0.224 & 0.276 & 0.548 & 0.852 & 0.558 & 0.457 & 0.680 & M 5.5 & M 6.0 & B \\
\hline J1917045-301920 & LP 924-17 & 1.33 & 0.253 & 0.279 & 0.533 & 0.855 & 0.594 & 0.486 & 0.720 & M 5.5 & M 5.5 & $\mathrm{~b}$ \\
\hline J2022480-564556 & $\ldots$ & 1.33 & 0.263 & 0.305 & 0.599 & 0.841 & 0.550 & 0.513 & 0.722 & M 5.5 & M 5.5 & A \\
\hline J2049527-171608 & LP $816-10$ & 1.48 & 0.219 & 0.288 & 0.548 & 0.806 & 0.500 & 0.435 & 0.620 & M 6.0 & M 6.0 & B \\
\hline $\mathrm{J} 2107247-335733$ & $\ldots$ & 1.34 & 0.251 & 0.302 & 0.626 & 0.851 & 0.548 & 0.495 & 0.692 & M 5.5 & M 5.5 & B \\
\hline $\mathrm{J} 2134222-431610$ & WT 792 & 1.32 & 0.293 & 0.292 & 0.592 & 0.833 & 0.574 & 0.531 & 0.712 & M 5.5 & M 5.0 & B \\
\hline J2202112-110945 & LP 759-17 & 1.53 & 0.194 & 0.302 & 0.596 & 0.769 & 0.453 & 0.397 & 0.520 & M 6.5 & M 6.0 & B \\
\hline $\mathrm{J} 2213504-634210$ & WT 887 & 1.29 & 0.215 & 0.237 & 0.488 & 0.856 & 0.576 & 0.464 & 0.733 & M 5.0 & M6.0 & B \\
\hline J2331217-274949 & $\ldots$ & 1.93 & 0.159 & 0.207 & 0.453 & 0.848 & 0.404 & 0.376 & 0.560 & M 8.5 & M7.0: & $\mathrm{B}$ \\
\hline $\mathrm{J} 2333405-213353$ & LHS 3970 & 1.31 & 0.227 & 0.306 & 0.620 & 0.860 & 0.577 & 0.506 & 0.699 & M 5.0 & M6.0 & B \\
\hline $\mathrm{J} 2337383-125027$ & LP 763-3 & 1.36 & 0.230 & 0.307 & 0.584 & 0.847 & 0.562 & 0.489 & 0.686 & M 5.5 & M 5.5 & B \\
\hline \multicolumn{13}{|l|}{ NTT objects: } \\
\hline J0351000-005244 & GJ 3252 & 1.62 & $0.251:$ & 0.307: & 0.654: & 0.745 & 0.437 & 0.451 & 0.592 & M 7.0 & M 5.5: & B \\
\hline J0436278-411446 & $\ldots$ & 1.88 & $0.226:$ & 0.282 : & 0.683: & 0.648 & 0.308 & 0.402 & 0.469 & M 8.0 & M 7.5: & $\mathrm{A}$ \\
\hline J0440231-053009 & LP 655-48 & 1.75 & $0.247:$ & 0.300: & 0.603: & 0.750 & 0.422 & 0.430 & 0.569 & M 7.5 & M7.5: & $\mathrm{b}$ \\
\hline J0518113-310153 & $\ldots$ & 1.49 & $0.216:$ & 0.273: & 0.602: & 0.755 & 0.448 & 0.413 & 0.597 & M 6.5 & M6.0: & A \\
\hline J0931223-171742 & LP 788-1 & 1.53 & $0.224:$ & $0.264:$ & $0.534:$ & 0.808 & 0.531 & 0.472 & 0.671 & M 6.5 & M 6.0: & $\mathrm{b}$ \\
\hline J1006319-165326 & LP 789-23 & 1.66 & $0.248:$ & 0.301: & 0.626 : & 0.743 & 0.429 & 0.448 & 0.585 & M 7.0 & M 5.5: & B \\
\hline J1048126-112009 & GJ 3622 & 1.60 & 0.183: & $0.227:$ & $0.521:$ & 0.787 & 0.475 & 0.437 & 0.617 & M 7.0 & M 6.0: & B \\
\hline J1155429-222458 & LP 851-346 & 1.75 & 0.199: & 0.248: & $0.546:$ & 0.753 & 0.423 & 0.421 & 0.557 & M 7.5 & M7.5: & B \\
\hline J1236396-172216 & $\ldots$ & 1.43 & 0.251: & 0.291: & $0.568:$ & 0.822 & 0.591 & 0.524 & 0.693 & M 6.0 & M 5.5: & A \\
\hline $\mathrm{J} 1250526-212113$ & $\ldots$ & 1.50 & $0.214:$ & 0.291: & 0.640: & 0.748 & 0.435 & 0.434 & 0.547 & M 6.5 & M 6.0: & B \\
\hline J2206227-204706 & $\ldots$ & 1.81 & 0.209: & $0.265:$ & 0.511: & 0.745 & 0.398 & 0.411 & 0.545 & M 8.0 & M7.5: & A \\
\hline $\mathrm{J} 2226443-750342$ & $\ldots$ & 1.91 & 0.290: & 0.329: & 0.600: & 0.733 & 0.379 & 0.452 & 0.524 & M 8.5 & M 8.0: & A \\
\hline
\end{tabular}

Columns 1 and 2: full DENIS name and other name.

Columns 3-10: spectroscopic indices. PC3 is defined in Martín et al. (1999); TiO5, CaH2 and CaH3 in Reid et al. (1995); VO1 in Hawley et al. (2002); VO2, TiO6 and TiO7 in Lépine et al. (2003).

Columns 11 and 12: spectral types derived from the (PC3, spectral type) relation of Martín et al. (1999) and from the (TiO5, spectral type) relation of Cruz \& Reid (2002), and rounded to the nearest half type. The types followed by “:” are unreliable, as discussed in the text, Sect. 2.1, as well as the NTT indices $\mathrm{TiO}, \mathrm{CaH} 2$ and $\mathrm{CaH} 3$.

Column 13: priority group (A, B or b), as defined in Sect. 2.1.

scale. As the denominator contains few data points, a good measurement of $\mathrm{TiO5}$ also requires a spectrum with significantly higher signal to noise ratio than PC3. On the astrophysical side, TiO5 is an excellent spectral type diagnostic for early $\mathrm{M}$ dwarfs, but it saturates and wraps around at spectral type M7. For late subtypes (e.g. J0306115-364753) the flux in the TiO5 numerator is also very low and the index therefore has large error bars except for very high $\mathrm{S} / \mathrm{N}$ spectra. It is therefore of limited value beyond $\sim \mathrm{M} 6$.
In our case, the resolution of NTT spectra is too low $(\sim 10 \AA)$; the TiO5 index is therefore not taken into account for them.

Figure 4 compares the PC3 and TiO5 indices for our T152 spectra, and demonstrates the saturation of TiO5 at late spectral types. Since our spectra have moderate signal to noise ratios but good relative flux calibration, we use the PC3 index as our main classification tool. We adopt the (Martín et al. 1999) 


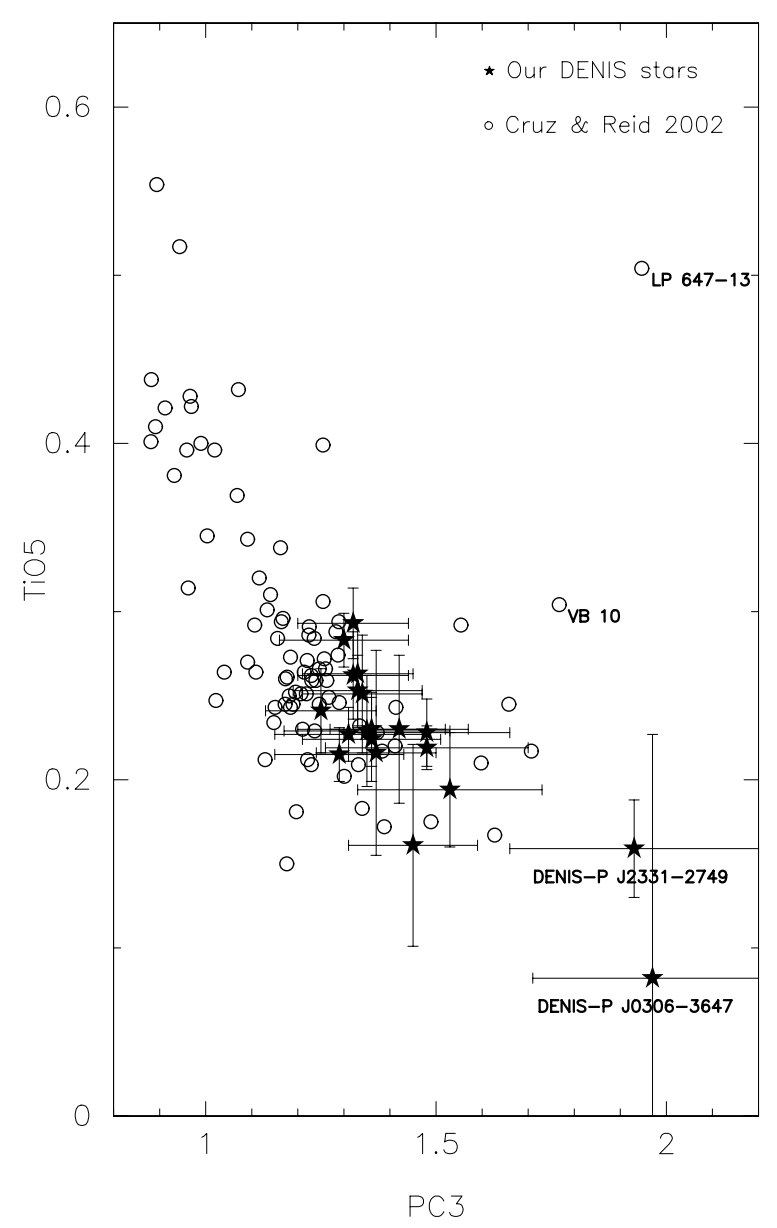

Fig. 4. TiO5 vs. PC3 diagram. The coolest stars are on the right side. The filled stars represent the $20 \mathrm{~T} 152$ measurements from this paper, and the empty circles represent measurements from Cruz \& Reid (2002). LP 647-13 (M9.0), VB 10 (flare star, M 8.0), DENISP J0306-3647 and DENIS-P J2331-2749 (both M 8.5 at 11 pc) are individually identified. The error bars are determined from the flux fluctuations over the integration intervals, and therefore represent lower limits to the true uncertainty. (The NTT stars are not plotted).

calibration of the PC3 index to spectral type, to determine the spectral class with an uncertainty of \pm 0.5 subclass.

For three new objects, DENIS-P J0306115-364753, DENIS-P J2226443-750342 and DENIS-P J2331217-274949 (hereafter DENIS-P J0306-3647, DENIS-P J2226-7503 and DENIS-P J2331-2749), the PC3 index indicates an M $8.5 \pm 0.5$ spectral type. Their spectral types from TiO5 would be about M 7, but the 3 spectra are evidently later than M 7. The stars are therefore simply beyond the validity range of the $\mathrm{TiO} 5$ calibration. Two of them are close neighbours of the Sun, both at $11 \pm 1.2 \mathrm{pc}$ (Sect. 4 ) and clearly of interest for parallax programs.

The CaH and VO indices (not plotted) do not reveal any subdwarfs. This is expected since our sample is mostly volumelimited, and too small to have much probability of including members of the stellar halo.

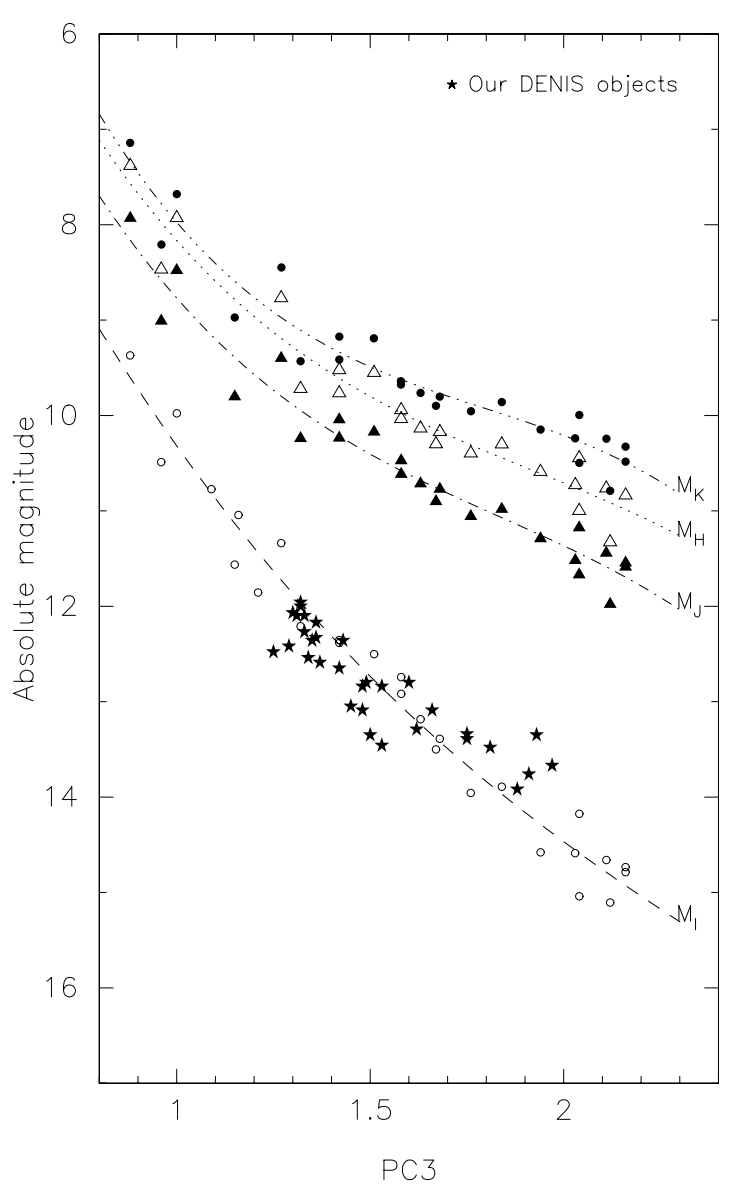

Fig. 5. Absolute magnitudes in the Cousins $I$ and CIT $J H K$ passbands as a function of PC 3 index (data in Table 2). The $M_{\mathrm{I}}$ values used for our objects are the ones derived from the DENIS $(I-J)$ photometry in Papers I and II and given again in Table 3, Col. 3.

\subsection{PC3 index-magnitude relation}

We identified in the literature 27 stars with good trigonometric parallaxes and photometry (Table 2) and with PC3 indices from Martín et al. (1999), Geballe et al. (2002) and Cruz \& Reid (2002), allowing to derive a calibration of absolute magnitudes in $I, J, H, K$ versus PC3 index (Phan-Bao 2002 contains an earlier version).

We transformed photometry from Leggett et al. (1998) and Weis (1996) to the Cousins-CIT systems, using the colour transformations published in the two papers. For our present purpose the DENIS system is close enough to Cousins-CTIO ( $\$ 0.05 \mathrm{mag}$, Delfosse 1997), and we therefore use the DENIS photometry without any corrections.

We excluded known doubles, unless the separation of the two stars is sufficient for individual photometric observations (e.g. Gl 720B, vB8, vB10), and all large amplitude variables. We did however have to accept a number of low amplitude flare stars, with peak visible light amplitude of 0.1 to $0.3 \mathrm{mag}$, since many very late $\mathrm{M}$ dwarfs have some photometric variability.

Table 2 summarizes the information, and Fig. 5 shows that the PC3 index tightly maps to absolute magnitudes in either of the $I, J, H$, and $K$ bands. The following cubic least-square fits 
Table 2. Red dwarfs with PC3 index, Cousins-CIT photometry and trigonometric parallaxes, used for the absolute magnitude calibration.

\begin{tabular}{|c|c|c|c|c|c|c|c|c|c|}
\hline $\begin{array}{l}\text { Stars } \\
\text { (1) }\end{array}$ & $\begin{array}{l}\alpha_{2000}, \delta_{2000} \\
(2)\end{array}$ & $\begin{array}{l}\text { Sp.T. } \\
\text { (3) }\end{array}$ & $\begin{array}{l}\text { PC3 } \\
\text { (4) }\end{array}$ & $\begin{array}{l}I \\
(5)\end{array}$ & $\begin{array}{l}I-J \\
(6)\end{array}$ & $\begin{array}{l}H \\
(7)\end{array}$ & $\begin{array}{l}K \\
(8) \\
\end{array}$ & $\begin{array}{l}\pi \\
(9)\end{array}$ & $\begin{array}{l}\text { References } \\
(10)\end{array}$ \\
\hline LHS 4 & $001825.79+440138.2$ & M 3.5 & 0.96 & 8.25 & 1.48 & 6.23 & 5.97 & $280.3 \pm 1.1$ & $2,6,6,9,9,9,9,16$ \\
\hline LP 292-67 & $002029.50+330505.5$ & M 5.5 & 1.21 & 12.36 & & $\ldots$ & $\cdots$ & $79.3 \pm 3.7$ & $2,8,7,15, \ldots, \ldots, \ldots, 17$ \\
\hline RG 0050-2722 & $005254.67-270559.5$ & M 9 & 2.16 & 16.67 & 3.19 & & 12.42 & $41.0 \pm 4.0$ & $1,5,5,1,1, \ldots, 1,17$ \\
\hline CTI $0126+28$ & $012739.13+280554.4$ & M 9 & 2.11 & 17.24 & 3.22 & 13.34 & 12.82 & $30.5 \pm 0.5$ & $4,5,5,11,11,11,11,11$ \\
\hline LHS 17 & $024614.97-045921.5$ & M 6 & 1.15 & 12.66 & 1.76 & $\ldots$ & 10.07 & $60.3 \pm 8.2$ & $1,8,7,1,1, \ldots, 1,17$ \\
\hline LP 412-31 & $032059.70+185422.7$ & M 8 & 1.84 & 14.70 & 2.91 & 11.11 & 10.67 & $68.9 \pm 0.6$ & $3,5,5,11,11,11,11,11$ \\
\hline LHS 2065 & $085336.11-032932.4$ & M 9 & 2.16 & 14.44 & 3.20 & 10.49 & 9.98 & $117.3 \pm 1.5$ & $2,5,5,9,9,9,9,17$ \\
\hline LHS 292 & $104812.64-112009.8$ & M 6.5 & 1.58 & 11.20 & 2.30 & 8.32 & 7.96 & $220.3 \pm 3.6$ & $1,6,6,9,9,9,9,17$ \\
\hline GJ 406 & $105628.99+070052.0$ & M 6 & 1.51 & 9.39 & 2.33 & 6.44 & 6.08 & $419.1 \pm 2.1$ & $2,5,5,9,9,9,9,17$ \\
\hline LHS 39 & $110530.31+433116.6$ & M 5.5 & 1.32 & 10.63 & 1.97 & 8.14 & 7.85 & $206.9 \pm 1.2$ & $2,6,6,9,9,9,9,16$ \\
\hline LHS 2397a & $112149.21-131308.3$ & M 8.5 & 2.04 & 14.95 & 3.00 & 11.22 & 10.77 & $70.0 \pm 2.1$ & $1,5,5,9,9,9,9,17$ \\
\hline BRI 1222-1221 & $122452.28-123835.2$ & M 8.5 & 1.94 & 15.74 & 3.29 & 11.75 & 11.31 & $58.6 \pm 3.8$ & $1,5,5,10,10,10,10,13$ \\
\hline LHS 330 & $122914.28+533305.0$ & M 6 & 1.42 & 14.38 & 2.15 & 11.76 & 11.41 & $39.9 \pm 1.0$ & $2,6,6,9,9,9,9,17$ \\
\hline LHS 2924 & $142843.33+331037.9$ & M 9 & 2.04 & 15.21 & 3.37 & 11.17 & 10.67 & $92.4 \pm 1.3$ & $2,5,5,9,9,9,9,17$ \\
\hline LHS 2930 & $143037.95+594324.1$ & M 6.5 & 1.68 & 13.31 & 2.62 & 10.09 & 9.72 & $103.8 \pm 1.3$ & $2,6,6,9,9,9,9,17$ \\
\hline LHS 3003 & $145638.38-280949.7$ & M 7 & 1.67 & 12.53 & 2.60 & 9.33 & 8.93 & $156.3 \pm 0.3$ & $2,6,6,9,9,9,9,17$ \\
\hline $\mathrm{T}^{*} 513-46546$ & $150108.19+225002.5$ & M 9 & 2.12 & 15.09 & 3.13 & 11.31 & 10.77 & $100.8 \pm 2.3$ & $4,5,5,12,12,12,12,17$ \\
\hline $\mathrm{T}^{*} 868-110639$ & $151016.86-024107.4$ & M 9 & 2.03 & 15.79 & 3.07 & 11.93 & 11.44 & $57.5 \pm 1.9$ & $1,5,5,12,12,12,12,17$ \\
\hline LHS 427 & $165525.32-081921.9$ & M 3.5 & 1.00 & 9.04 & 1.50 & 6.99 & 6.74 & $154.0 \pm 4.0$ & $2,6,6,9,9,9,9,16$ \\
\hline VB 8 & $165535.35-082342.3$ & M 7 & 1.63 & 12.24 & 2.47 & 9.19 & 8.82 & $154.5 \pm 0.7$ & $2,8,7,9,9,9,9,18$ \\
\hline LHS 3339 & $175533.50+582426.5$ & M 6 & 1.42 & 14.02 & 2.31 & 11.19 & 10.84 & $46.4 \pm 1.0$ & $2,6,6,10,10,10,10,17$ \\
\hline GJ 1224 & $180732.88-155746.8$ & M 4.5 & 1.16 & 10.43 & $\ldots$ & $\ldots$ & $\ldots$ & $132.6 \pm 3.7$ & $2,8,7,15, \ldots, \ldots, \ldots, 17$ \\
\hline GJ 1227 & $182227.28+620300.8$ & M 4.5 & 1.09 & 10.35 & $\ldots$ & $\ldots$ & $\ldots$ & $121.5 \pm 2.2$ & $2,8,7,14, \ldots, \ldots, \ldots, 17$ \\
\hline Gl 720B & $183527.39+454539.6$ & M 3.5 & 0.88 & 10.32 & 1.44 & 8.33 & 8.09 & $64.6 \pm 1.0$ & $2,8,7,9,9,9,9,16$ \\
\hline VB 10 & $191657.66+050900.4$ & M 8 & 1.76 & 12.80 & 2.90 & 9.24 & 8.80 & $170.3 \pm 1.4$ & $2,5,5,9,9,9,9,16$ \\
\hline LHS 523 & $222854.38-132517.8$ & M 6.5 & 1.58 & 13.00 & 2.27 & 10.20 & 9.90 & $88.8 \pm 4.9$ & $1,6,6,9,9,9,9,17$ \\
\hline GJ 905 & $234155.17+441038.0$ & M 5 & 1.27 & 8.84 & 1.94 & 6.27 & 5.95 & $316.0 \pm 1.1$ & $2,5,5,9,9,9,9,17$ \\
\hline
\end{tabular}

T*: TVLM

Columns 1 and 2: object name and coordinates for equinox and epoch J2000.

Columns 3 and 4: spectral type and PC3 index.

Columns 5-8: optical and infrared photometry in the Cousins-CIT systems.

Column 9: trigonometric parallax and its standard error, in mas.

Column 10: references for all parameters listed in the Cols. 2-9, column by column: 1) DENIS; 2) Bakos et al. (2002); 3) Salim \& Gould (2003); 4) Monet et al. (2003); 5) Martín et al. (1999); 6) Geballe et al. (2002); 7) Cruz \& Reid (2002); 8) Hawley et al. (1996); 9) Leggett (1992); 10) Leggett et al. (1998); 11) Dahn et al. (2002); 12) Tinney et al. (1993); 13) Tinney (1996); 14) Bessell (1990); 15) Weis (1996); 16) HIP (ESA 1997); 17) GCTP (van Altena et al. 1995); 18) Monet et al. (1992).

to those data:

$$
\begin{aligned}
M_{I}= & 2.227+10.871(\mathrm{PC} 3)-3.185(\mathrm{PC} 3)^{2} \\
& +0.4048(\mathrm{PC} 3)^{3} \\
M_{J}= & -0.5630+15.384(\mathrm{PC} 3)-7.396(\mathrm{PC} 3)^{2} \\
& +1.343(\mathrm{PC} 3)^{3} \\
M_{H}= & -0.7493+14.408(\mathrm{PC} 3)-6.647(\mathrm{PC} 3)^{2} \\
& +1.154(\mathrm{PC} 3)^{3} \\
M_{K}= & -2.877+18.779(\mathrm{PC} 3)-9.738(\mathrm{PC} 3)^{2} \\
& +1.810(\mathrm{PC} 3)^{3}
\end{aligned}
$$

are valid for $0.9 \leq \mathrm{PC} 3 \leq 2.2$, or spectral types between M 3.5 and $\mathrm{M} 9$. Over this range the rms dispersion of the data around the fits is approximately $0.25 \mathrm{mag}$, in any of the 4 photometric bands. This corresponds to a $\sim 12 \%$ standard error on distances to single stars.

\subsection{Spectral types and distances}

As discussed above, the (PC3 vs. spectral type) relation of Martín et al. (1999) is our main spectral classification tool for the M dwarfs, and we use the TiO5 vs. spectral type relation of Cruz \& Reid (2002) as a cross-check. The two relations produce consistent spectral types, except beyond the $\sim$ M 6 validity limit of TiO5. In Table 3 we therefore only list the PC3 spectral types. The table also lists the absolute magnitudes in the 3 DENIS bands computed from the PC3 index, as well as the estimated spectrophotometric distances for each of those bands and their average. The values for the 3 colours $I, J, K$ are very similar, indicating correlated uncertainties for the 3 estimators. As customary, the distances and uncertainties assume that all stars are single, while some fraction will in fact be unresolved binaries and will have their distances underestimated by up to $\sqrt{2}$. Table 3 with its improved distances contains 10 new stars closer than $15 \mathrm{pc}$, of which 2 are closer than $12 \mathrm{pc}$ and one closer than $10 \mathrm{pc}$. 
Table 3. Spectrophotometric distance and spectral type for the 32 nearby $M$ dwarfs.

\begin{tabular}{|c|c|c|c|c|c|c|c|c|c|c|c|c|c|c|}
\hline Stars & (2) & $\begin{array}{l}I-J \\
(3) \\
\end{array}$ & $\begin{array}{l}J-K \\
(4) \\
\end{array}$ & $\begin{array}{l}\text { PC3 } \\
\text { (5) } \\
\end{array}$ & $\begin{array}{l}M_{I} \\
(I J) \\
(6) \\
\end{array}$ & $\begin{array}{l}M_{I} \\
(\mathrm{PC} 3) \\
(7) \\
\end{array}$ & $\begin{array}{l}M_{J} \\
(\mathrm{PC} 3) \\
(8) \\
\end{array}$ & $\begin{array}{l}M_{K} \\
(\mathrm{PC} 3) \\
(9) \\
\end{array}$ & $\begin{array}{l}d_{I} \\
(\mathrm{PC} 3) \\
(10) \\
\end{array}$ & $\begin{array}{l}d_{J} \\
(\mathrm{PC} 3) \\
(11) \\
\end{array}$ & $\begin{array}{l}d_{K} \\
(\mathrm{PC} 3) \\
(12) \\
\end{array}$ & $\begin{array}{l}d_{\mathrm{sp}} \\
(\mathrm{PC} 3) \\
(13) \\
\end{array}$ & $\begin{array}{l}d \\
(I J) \\
(14) \\
\end{array}$ & $\begin{array}{l}\text { Sp. } \\
\text { type } \\
(15) \\
\end{array}$ \\
\hline J0004-17 & 13.00 & 2.03 & 0.93 & 1.32 & 12.00 & 11.96 & 9.95 & 9.11 & 16.2 & 16.0 & 15.4 & 15.9 & 15.9 & M 5.5 \\
\hline J0013-00 & 14.37 & 2.22 & 0.88 & 1.37 & 12.59 & 12.18 & 10.08 & 9.23 & 27.4 & 25.9 & 25.6 & 26.3 & 22.7 & M 5.5 \\
\hline J0013-04 & 13.88 & 2.44 & 0.99 & 1.48 & 13.09 & 12.65 & 10.36 & 9.45 & 17.6 & 16.5 & 15.8 & 16.6 & 14.4 & M 6.0 \\
\hline J0019-36 & 14.30 & 2.14 & 0.89 & 1.35 & 12.36 & 12.09 & 10.03 & 9.18 & 27.6 & 26.7 & 26.2 & 26.8 & 24.4 & M 5.5 \\
\hline J0100-61 & 15.01 & 2.42 & 0.94 & 1.45 & 13.05 & 12.53 & 10.29 & 9.40 & 31.4 & 28.9 & 28.2 & 29.5 & 24.6 & M 6.0 \\
\hline J0306-36 & 14.41 & 2.79 & 1.03 & 1.97 & 13.67 & 14.38 & 11.31 & 10.16 & 10.2 & 11.5 & 12.2 & 11.3 & 14.0 & M 8.5 \\
\hline J0351-00 & 13.75 & 2.55 & 0.99 & 1.62 & 13.29 & 13.20 & 10.66 & 9.68 & 12.9 & 12.8 & 12.7 & 12.8 & 12.4 & M 7.0 \\
\hline J0436-41 & 16.04 & 2.92 & 1.12 & 1.88 & 13.92 & 14.10 & 11.14 & 10.04 & 24.5 & 24.9 & 24.7 & 24.7 & 26.6 & M 8.0 \\
\hline J0440-05 & 13.35 & 2.61 & 1.19 & 1.75 & 13.39 & 13.67 & 10.91 & 9.86 & 8.6 & 9.3 & 8.7 & 8.9 & 9.8 & M 7.5 \\
\hline J0518-31 & 14.17 & 2.30 & 1.00 & 1.49 & 12.80 & 12.69 & 10.38 & 9.47 & 19.7 & 19.8 & 19.0 & 19.5 & 18.8 & M 6.5 \\
\hline J0931-17 & 13.36 & 2.32 & 1.01 & 1.53 & 12.84 & 12.85 & 10.47 & 9.54 & 12.6 & 13.0 & 12.5 & 12.7 & 12.7 & M 6.5 \\
\hline J1006-16 & 14.55 & 2.44 & 1.14 & 1.66 & 13.09 & 13.35 & 10.74 & 9.74 & 17.4 & 18.8 & 17.6 & 17.9 & 19.6 & M 7.0 \\
\hline J1048-11 & 11.25 & 2.30 & 0.98 & 1.60 & 12.80 & 13.13 & 10.62 & 9.65 & 4.2 & 4.6 & 4.6 & 4.5 & 4.9 & M 7.0 \\
\hline J1155-22 & 13.48 & 2.58 & 1.05 & 1.75 & 13.34 & 13.67 & 10.91 & 9.86 & 9.2 & 10.0 & 9.9 & 9.7 & 10.7 & M 7.5 \\
\hline J1236-17 & 13.91 & 2.14 & 1.14 & 1.43 & 12.36 & 12.44 & 10.24 & 9.36 & 19.6 & 20.2 & 18.0 & 19.3 & 20.4 & M 6.0 \\
\hline $\mathrm{J} 1250-21$ & 13.78 & 2.59 & 1.11 & 1.50 & 13.35 & 12.73 & 10.40 & 9.49 & 16.2 & 14.4 & 13.1 & 14.6 & 12.2 & M 6.5 \\
\hline J1538-10 & 14.36 & 2.18 & 0.95 & 1.25 & 12.48 & 11.63 & 9.73 & 8.92 & 35.2 & 30.8 & 29.0 & 31.7 & 23.8 & M 5.0 \\
\hline J1552-26 & 12.61 & 2.24 & 1.07 & 1.42 & 12.65 & 12.40 & 10.21 & 9.34 & 11.0 & 10.7 & 9.8 & 10.5 & 9.8 & M 6.0 \\
\hline J1553-23 & 13.64 & 2.05 & 1.02 & 1.30 & 12.07 & 11.87 & 9.89 & 9.06 & 22.6 & 21.9 & 20.1 & 21.5 & 20.6 & M 5.0 \\
\hline J1610-06 & 13.46 & 2.08 & 1.09 & 1.36 & 12.17 & 12.14 & 10.06 & 9.20 & 18.4 & 18.4 & 16.5 & 17.7 & 18.1 & M 5.5 \\
\hline J1917-30 & 13.81 & 2.11 & 0.95 & 1.33 & 12.27 & 12.00 & 9.97 & 9.13 & 23.0 & 22.1 & 21.1 & 22.1 & 20.3 & M 5.5 \\
\hline J2022-56 & 13.81 & 2.06 & 0.80 & 1.33 & 12.10 & 12.00 & 9.97 & 9.13 & 23.0 & 22.7 & 23.1 & 22.9 & 22.0 & M 5.5 \\
\hline J2049-17 & 14.16 & 2.32 & 1.08 & 1.48 & 12.84 & 12.65 & 10.36 & 9.45 & 20.0 & 19.8 & 18.3 & 19.4 & 18.3 & M 6.0 \\
\hline J2107-33 & 14.36 & 2.20 & 1.02 & 1.34 & 12.54 & 12.05 & 10.00 & 9.16 & 29.0 & 27.0 & 24.9 & 27.0 & 23.1 & M 5.5 \\
\hline J2134-43 & 12.78 & 2.02 & 1.06 & 1.32 & 11.96 & 11.96 & 9.95 & 9.11 & 14.6 & 14.5 & 13.1 & 14.1 & 14.6 & M 5.5 \\
\hline J2202-11 & 15.11 & 2.66 & 0.98 & 1.53 & 13.46 & 12.85 & 10.47 & 9.54 & 28.3 & 24.9 & 24.3 & 25.8 & 21.3 & M 6.5 \\
\hline J2206-20 & 15.09 & 2.67 & 1.22 & 1.81 & 13.48 & 13.87 & 11.02 & 9.94 & 17.5 & 19.1 & 17.8 & 18.2 & 21.0 & M 8.0 \\
\hline $\mathrm{J} 2213-63$ & 13.05 & 2.16 & 1.04 & 1.29 & 12.42 & 11.82 & 9.86 & 9.03 & 17.6 & 16.1 & 14.6 & 16.1 & 13.3 & M 5.0 \\
\hline $\mathrm{J} 2226-75$ & 15.20 & 2.84 & 1.20 & 1.91 & 13.76 & 14.19 & 11.20 & 10.08 & 15.9 & 17.1 & 16.5 & 16.5 & 19.4 & M 8.5 \\
\hline $\mathrm{J} 2331-27$ & 14.25 & 2.59 & 1.04 & 1.93 & 13.35 & 14.25 & 11.23 & 10.11 & 10.0 & 12.2 & 12.7 & 11.6 & 15.1 & M 8.5 \\
\hline J2333-21 & 13.89 & 2.06 & 0.90 & 1.31 & 12.10 & 11.91 & 9.92 & 9.08 & 24.9 & 24.1 & 23.4 & 24.1 & 22.8 & M 5.0 \\
\hline $\mathrm{J} 2337-12^{a}$ & 13.67 & 2.13 & 1.10 & 1.36 & 12.33 & 12.14 & 10.06 & 9.20 & 20.2 & 19.8 & 17.7 & 19.2 & 18.5 & M 5.5 \\
\hline
\end{tabular}

${ }^{a}$ Also discussed in Cruz \& Reid (2002).

Column 1: abbreviated DENIS name.

Columns 2-4: DENIS photometry; Col. 5: PC3 index.

Column 6: absolute $I$-band magnitude derived in Paper II from the $I-J$ colour.

Column 7-9: absolute magnitudes for the $I, J, K$ bands based on the PC3-absolute magnitudes relation.

Columns 10-12: distance (pc) estimated from the DENIS photometry and the $M_{I}, M_{J}, M_{K}$ derived from the PC3 index.

Columns 13 and 14: adopted distance, and distance previously derived in Paper II from the the $I-J$ colour.

Column 15: spectral types derived from the PC3 index, rounded to the nearest half subtype.

The star lists observed at T152 and NTT are merged and ordered by increasing RA.

\section{Discussion}

\subsection{Distant reddened main sequence stars}

Three of the four reddened main sequence stars (DENIS-P J1552237-033520, DENIS-P J1553186-025919, and DENISP J1553251-044741) belong to our main sample, while DENIS-P J1625503-240008 = LP 862-26 is in the extended sample. All four stars passed our reduced proper motion selection (Paper II), and two have moderately high proper motions, 0.13 and $0.16 \operatorname{arcsec} \mathrm{yr}^{-1}$. We expected both to be nearby $M$ dwarfs, and their identification was a surprise to us, since we had restricted our selection to high galactic latitudes $\left(\left|b_{\text {III }}\right|>20\right.$ degrees) and eliminated objects superimposed on large molecular complexes such as Upper Scorpius. That selection turns out to have been insufficiently stringent, and all $4 \mathrm{ob}-$ jects are in the background of known high latitude molecular clouds, which had escaped our detection: J1552-03, J1553-04 and J1553-02 are in the vicinity of the well-known Lynds 134 and Lynds 183 (also known as Lynds 134N) dark nebulae, and J1625-24 is in the background of the Rho Ophiucus molecular complex. Our cleaning procedure clearly needs to include smaller molecular clouds than we had initially expected. Aside from this easily-corrected shortcoming, our reduced proper motion selection proves very discriminating: with an updated 


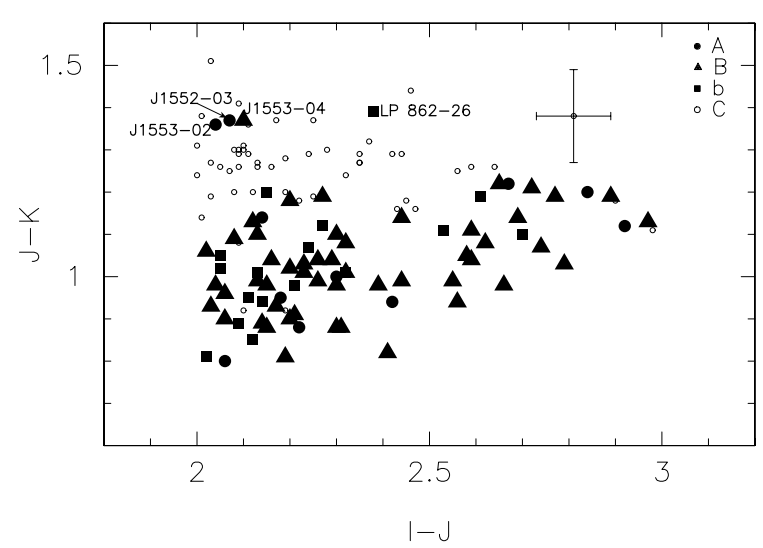

Fig. 6. DENIS $(I-J, J-K)$ colour-colour diagram of the 132 objects in the full sample. A, B and b groups = suspected $\mathrm{M}$ dwarfs, as defined in Sect. 2.1; $\mathrm{C}=$ suspected giants. The 4 objects at the top of the diagram are the strongly reddened early type stars located behind molecular clouds; there are no other dwarf candidates in that part of the colourcolour diagram. The cross indicates representative error bars.

mask for molecular clouds, all observed targets match their expected status.

Since we obtained spectra for only a fraction of the nearby star candidates, one legitimate concern is whether the full sample contains additional reddened early-type contaminants. Fortunately, the $(I-J, J-K)$ colour-colour diagram of the full sample (Fig. 6) indicates that this is very unlikely. At a given $I-J$ colour, the giant candidates have a significantly larger $J-K$ colour than the dwarf candidates. At the precision of the DENIS photometry the two distributions overlap to some small extent around $I-J=1.2$, making that diagram an imperfect dwarf/giant diagnostic tool (see Paper I, Fig. 2). The 4 reddened stars, on the other hand, have even higher $J-K$ colours than any of the giants. They stand well clear of any of the nearby dwarf candidates, and we can thus safely conclude that the M dwarf lists of Papers I and II contain no other reddened object.

\subsection{Low proper motion nearby $M$ dwarfs}

After excluding the reddened early type stars, our main sample of 62 dwarf candidates still contains 59 nearby dwarf candidates. 27 of them were observed, including all those with a proper motion below $0.1 \operatorname{arcsec}_{\mathrm{yr}}{ }^{-1}$. As discussed above, it is unlikely that any of the unobserved stars is a contaminant. Of those 59 dwarfs, $9(15 \%)$ have a proper motion below $0.1 \operatorname{arcsec} \mathrm{yr}^{-1}$ and a further $7(11 \%)$ between this value and the 0.18 arcsec $\mathrm{yr}^{-1}$ threshold of the NLTT, for a total of $26 \%$. This suggests that proper motion selection with traditional thresholds may miss up to a quarter of the $d<30 \mathrm{pc}$ population. Photometric binaries and the Malmquist bias will however both move some of those stars beyond $30 \mathrm{pc}$, making this a moderate overestimate.

Our main sample of A and B stars should be complete up to about $30 \mathrm{pc}$ over the 5700 square degrees, as explained in Papers I and II. In order to better evaluate the fraction of "slow stars" to be expected versus distance, counts were performed in the HIPPARCOS Catalogue (ESA 1997). Of course it would

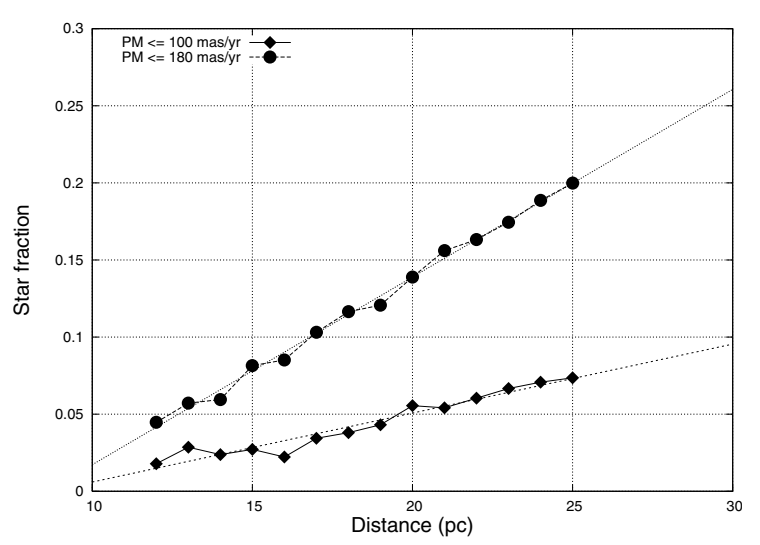

Fig. 7. Fractions of HIP stars brighter than $M_{V}=9$ and with low PM

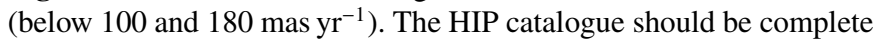
for them. The linear fits can be extrapolated up to $30 \mathrm{pc}$.

have been possible to use some kinematical model with dispersions varying with the direction according to the velocity ellipsoid; but the counts are together very simple and quite accurate, and are therefore much more reliable and preferable. All bright enough ( $V \leq 11.5)$, known or suspected, nearby stars were included in the Hipparcos Catalogue. For $25 \mathrm{pc}$ this corresponds to $M_{V}=9.5$. On the other hand, the CNS3 (Gliese \& Jahreiss, 1991) is considered as complete to $M_{V}=9$; therefore all stars closer than $25 \mathrm{pc}$ and brighter than $M_{V}=9$ should be in HIP and can be counted with their HIP distance and proper motion. Figure 7 shows the fraction of HIP stars

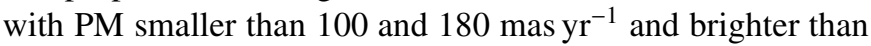
$M_{V}=9$ vs. distance. Although the correct analytical shape of these curves is not known with accuracy, clearly a linear fit can be made and safely extrapolated up to $30 \mathrm{pc}$. The fractions of stars to be expected up to 30 pc with PM below 100 and $180 \mathrm{mas} \mathrm{yr}^{-1}$ are respectively 10 and $26 \%$, not including the Poisson noise. Our observed fraction of stars with PM below

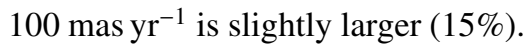

\section{Summary}

Table 4 summarizes the distribution of stars according to initial categories and final classification. Within the 5700 square degrees statistical sample, 3 of the 62 dwarf candidates are distant main sequence stars reddened by well known molecular clouds, and are easily recognisable from both their $J-K$ colour and their position on the sky. With hindsight they could easily have been eliminated before spectroscopic observations. The others are all confirmed as late-type members of the immediate solar neighborhood. 9 of them have low proper motions, below $0.1 \operatorname{arcsec} \mathrm{yr}^{-1}$. This $100 \%$ success rate demonstrates that photometry and Reduced Proper Motion alone can be used to reliably identify members of the immediate solar neighbourhood. Spectroscopy obviously provides additional characterisation, but is not strictly needed for confirmation.

One quarter of the 59 members of our statistically welldefined sample of stars with a photometric distance below $30 \mathrm{pc}$ over 5700 square degrees has a proper motion below the NLTT threshold. 
Table 4. Sample summary.

\begin{tabular}{|c|c|c|c|c|c|c|}
\hline $\begin{array}{l}\text { Prio. group, } \\
\text { as in Sect. } 2.1\end{array}$ & $\overline{\mathrm{A}}$ & $\overline{\mathrm{B}}$ & $\mathrm{A}+\mathrm{B}$ & $\overline{\mathrm{C}}$ & $\bar{b}$ & Total \\
\hline $\begin{array}{c}\text { Number of } \\
\text { stars in } \\
\text { Papers I and II }\end{array}$ & 11 & 51 & 62 & 52 & 18 & 132 \\
\hline $\begin{array}{l}\text { Observed, } \\
\text { this paper }\end{array}$ & 11 & 19 & 30 & 3 & 6 & 39 \\
\hline $\begin{array}{c}\text { Classification } \\
\text { after } \\
\text { observation }\end{array}$ & $\begin{array}{l}9 \mathrm{DW} \\
+2 \mathrm{R}\end{array}$ & $\begin{array}{c}18 \mathrm{Dw} \\
+1 \mathrm{R}\end{array}$ & $\begin{array}{c}27 \mathrm{Dw} \\
+3 \mathrm{R}\end{array}$ & $3 \mathrm{G}$ & $\begin{array}{l}5 \mathrm{Dw} \\
+1 \mathrm{R}\end{array}$ & $\begin{array}{l}32 \mathrm{Dw} \\
+4 \mathrm{R} \\
+3 \mathrm{G} \\
\end{array}$ \\
\hline
\end{tabular}

Columns A, B, $(\mathrm{A}+\mathrm{B})$ and $\mathrm{C}$ represent the contents of the main sample, over the 5700 square degrees;

Column "b" represents the extended sample (only NLTT stars), and is not taken into account for the statistics.

Last line: "Dw" stands for "Dwarfs"; "R" for "reddened star"; "G" for "giant".

We calibrated the PC3 spectral index to absolute magnitudes, and used it to derive spectrophotometric distance to the 32 late-type $\mathrm{M}$ dwarfs. Three objects have nominal distances within 12 pc: two new M 8.5 dwarfs at 11 pc (DENIS-P J0306-3647 and DENIS-P J2331-2749), as well as one previously known (but without distance) M 7.5 dwarf at 10 pc (DENIS-P J1552446-262313, LP 860-41).

Acknowledgements. The authors acknowledge help during the observations by the $2 \mathrm{p} 2$ team at the European Southern Observatory, John Pritchard, Ivo Saviane and Rolando Vega. We also thank the DENIS consort for both data and good advice.The referee's comments were very useful for many improvements and clarifications. P.-B.N. acknowlegdes financial support from Vietnamese research grant B2004-09-13, and a nice Ph.D. stay at CAI, Observatoire de Paris. E.M. acknowledges financial support from NSF grant AST 0205862.

This research has made use of the SIMBAD and VIZIER databases, operated at CDS, Strasbourg, France.

\section{References}

Bakos, G. A., Sahu, K. C., \& Németh, P. 2002, ApJS, 141, 187 Bessell, M. S. 1990, A\&AS, 83, 357

Cruz, K. L., \& Reid, I. N. 2002, AJ, 123, 2828

Dahn, C. C., Harris, H. C., Vrba, F. J., et al. 2002, AJ, 124, 1170

Delfosse, X. 1997, Ph.D. Thesis, Grenoble University

Epchtein, N. 1997, in the 2nd DENIS Euroconference, The impact of large scale near-infrared surveys, ed. F. Garzon, et al. (Kluwer Dordrecht), 15

ESA 1997, The Hipparcos and Tycho Catalogues, ESA SP-1200

Geballe, T. R., Knapp, G. R., Leggett, S. K., et al. 2002, ApJ, 564, 466

Gliese, W., \& Jahreiss, H. 1991, Catalogue of Nearby Stars III, file available at CDS

Hawley, S. L., Gizis, J. E., \& Reid, I. N. 1996, AJ, 112, 2799

Hawley, S. L., Covey, K. R., Knapp, G. R., et al. 2002, AJ, 123, 3409

Leggett, S. K. 1992, ApJS, 82, 531

Leggett, S. K., Allard, F., \& Hauschildt, P. H. 1998, ApJ, 509, 836

Lépine, S., Rich, R. M., \& Shara, M. M. 2003, AJ, 125, 1598

Luyten, W. J. 1979, Catalogue of stars with proper motions exceeding 0.' 5 annually (LHS) (Minneapolis: University of Minnesota)

Luyten, W. J. 1980, New Luyten catalog of stars with proper motions larger than Two Tenths of an arcsecond (NLTT) (Minneapolis: University of Minnesota)

Martín, E. L., Delfosse, X., Basri, G., et al. 1999, AJ, 118, 2466

Monet, D. G., Dahn, C. C., Vrba, F. J., et al. 1992, AJ, 103, 638

Monet, D. G., Levine, S. E., Canzian, B., et al. 2003, AJ, 125, 984

Phan-Bao, N., Guibert, J., Crifo, F., et al. 2001, A\&A, 380, 590

Phan-Bao, N. 2002, Ph.D. Thesis, Very low mass stars in the solar neighbourhood, Paris Observatory

Phan-Bao, N., Crifo, F., Delfosse, X., et al. 2003, A\&A, 401, 959

Phan-Bao, N., Martín, E. L., Reylé, C., Forveille, T., \& Lim, J. 2005, A\&A, 439, L19

Reid, I. N., Hawley, S. L., \& Gizis, J. E. 1995, AJ, 110, 1838

Reid, I. N. 2003, AJ, 126, 2449

Salim, S., \& Gould, A. 2003, ApJ, 582, 1011

Tinney, C. G., Mould, J. R., \& Reid, I. N. 1993, AJ, 105, 1045

Tinney, C. G. 1996, MNRAS, 281, 644

van Altena, W. F., Lee, J. T., \& Hoffleit, E. D. 1995, The General Catalogue of Trigonometric Stellar Parallaxes, Fourth edition (New Haven, CT: Yale University Observatory)

Weis, E. W. 1996, AJ, 112, 2300

Vyssotsky, A. N. 1943, ApJ, 97, 381

Vyssotsky, A. N. 1946, ApJ, 104, 234

Vyssotsky, A. N. 1952, ApJ, 116, 117 